\title{
Novel Mode of Antagonist Binding in NMDA Receptors Revealed by the Crystal Structure of the GluN1-GluN2A Ligand-Binding Domain Complexed to NVP-AAM077 ${ }^{\mathrm{s}}$
}

\section{Annabel Romero-Hernandez and Hiro Furukawa}

\author{
Keck Structural Biology Laboratory, Cold Spring Harbor Laboratory, Cold Spring Harbor, New York (A.R.-H., H.F.); and Watson \\ School of Biological Sciences, Cold Spring Harbor, New York (A.R.-H., H.F.)
}

Received December 15, 2016; accepted May 1, 2017

\begin{abstract}
Competitive antagonists against $N$-methyl-D-aspartate (NMDA) receptors have played critical roles throughout the history of neuropharmacology and basic neuroscience. There are currently numerous NMDA receptor antagonists containing a variety of chemical groups. Among those compounds, a GluN2-specific antagonist, (R)-[(S)-1-(4-bromo-phenyl)-ethylamino]-(2,3-dioxo1,2,3,4-tetrahydroquinoxalin-5-yl)-methyl-phosphonic acid (NVP-AAM077), contains a unique combination of a dioxoquinoxalinyl ring, a bromophenyl group, and a phosphono group. In this study, we present the crystal structure of the isolated ligand-binding domain of the GluN1-GluN2A NMDA receptor in
\end{abstract}

complex with the GluN1 agonist glycine and the GluN2A antagonist NVP-AAM077. The structure shows placement of the dioxoquinoxalinyl ring and the phosphono group of NVP-AAM077 in the glutamate-binding pocket in GluN2A and the novel interaction between the bromophenyl group and GluN1-Glu781 at the GluN1-GluN2A subunit interface. Site-directed mutagenesis of GluN1-Glu781 reduced the potency of inhibition by NVP-AAM077, thus confirming the involvement of the GluN1 subunit for binding of NVP-AAM077. The unique antagonist-binding pattern shown in this study provides a novel dimension to design and create antagonists with potential therapeutic values.

\section{Introduction}

Antagonists against $N$-methyl-D-aspartate (NMDA) receptors have played critical roles to delineate the physiologic roles of NMDA receptors in brain functions and in neurologic disorders and diseases in many studies. NMDA receptors belong to the large family of ionotropic glutamate receptors (iGluRs) that mediate the majority of fast excitatory synaptic transmission in the mammalian brain. Dysfunctional NMDA receptors have been implicated in various neurologic disorders and diseases, including schizophrenia, depression, Alzheimer's disease, stroke, and seizure. Thus, in addition to applications in basic neuroscience research, NMDA receptor antagonists have drawn significant

This work was supported by the National Institutes of Health [Grants MH085926 and GM105730], the Stanley Institute of Cognitive Genomics, and the Robertson Research Fund of Cold Spring Harbor Laboratory (to H.F.). A.R.-H. was funded by the Genentech Foundation Fellowship and the Starr Foundation.

https://doi.org/10.1124/mol.116.107912.

S This article has supplemental material available at molpharm. aspetjournals.org. attention as potential therapeutic reagents. The effort to develop competitive NMDA receptor antagonists started from $\mathrm{D}$ - $\alpha$-aminoadipate (McLennan and Lodge, 1979) with moderate specificity and potency, leading to the significantly more potent NMDA-selective compound, D-(-)-2-amino-5-phosphonopentanoic acid (D-AP5) (Evans et al., 1982) containing the amino acid backbone and a phosphono group. This was followed by compounds with phenanthrene backbones such as 1-(phenanthrene-2carbonyl)-piperazine-2, 3-dicarboxylic acid (PPDA) (Feng et al., 2004). Furthermore, another compound, (R)-[(S)-1-(4-bromo-phenyl)ethylamino]-(2,3-dioxo-1,2,3,4-tetrahydroquinoxalin-5-yl)-methylphosphonic acid (NVP-AAM077), with an anticonvulsant effect in animals became available (Auberson et al., 2002) (Fig. 1A). NVP-AAM077 was originally reported to have over 100 -fold specificity toward the GluN1-GluN2A NMDA receptors over GluN1-GluN2B NMDA receptors but was later shown to have only fivefold specificity through more accurate and extensive pharmacological analyses (Frizelle et al., 2006).

NMDA receptors, like any other iGluR family members, are heterotetrameric ion channels composed of two GluN1

ABBREVIATIONS: AMPA, $\alpha$-amino-3-hydroxy-5-methyl-4-isoxazole propionic acid; ATD, amino-terminal domain; D-AP5, D-(-)-2-amino-5phosphonopentanoic acid; iGluR, ionotropic glutamate receptor; LBD, ligand-binding domain; LLG, Log-likelihood gain; NAM, negative allosteric modulator; NMDA, N-methyl-D-aspartate; NVP-AAM077, (R)-[(S)-1-(4-bromo-phenyl)-ethylamino]-(2,3-dioxo-1,2,3,4-tetrahydroquinoxalin-5-yl)methyl-phosphonic acid; PPDA, 1-(phenanthrene-2-carbonyl)-piperazine-2, 3-dicarboxylic acid; RFZ, Rotation function Z-score; TFZ, translation function Z-score; TMD, transmembrane domain; LLG, Log- Likelihood Gain; RFZ, Rotation function Z-score; TFZ, Translation function Z-score. 

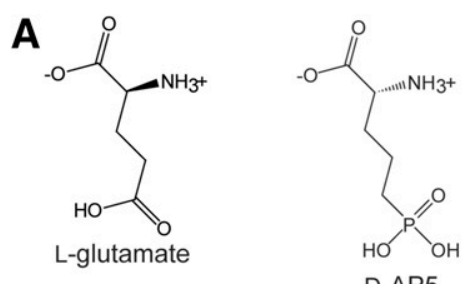

D-AP5
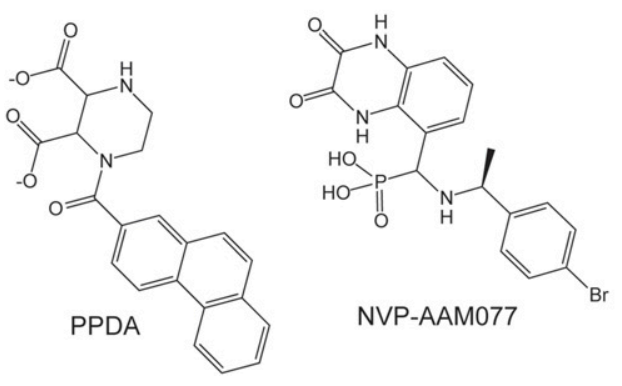

NVP-AAM077
B

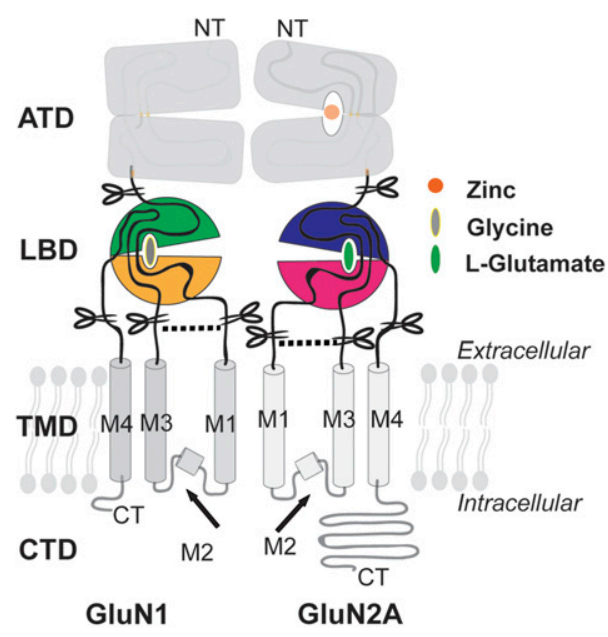

Fig. 1. Ligands and domain organization of NMDA receptors. (A) Chemical structures of the agonist glutamate, and competitive antagonists, D-AP5, PPDA, and NVP-AAM077. (B) Domain organization of NMDA receptor subunits, which are composed of an ATD, an ligand-binding domain (LBD), a TMD, and a carboxy-terminal domain (CTD). The LBD is isolated by tethering the two peptides separated by the M1 and M3 TMD helices by a Gly-Thr dipeptide linker (dots). NT and CT stand for the amino- terminus and the carboxy-terminus, respectively. subunits and two GluN2 and/or GluN3 subunits (Traynelis et al., 2010). The eight splice variants of the GluN1 subunits (1a-4a and $1 \mathrm{~b}-4 \mathrm{~b})$ and the two GluN3 subunits (A-B) bind to glycine, whereas the four GluN2 subunits (A-D) bind to glutamate or NMDA. All of the above subunits contain modular domains, including an amino-terminal domain (ATD), a ligand-binding domain (LBD), a transmembrane domain (TMD), and a carboxyl-terminal domain (Fig. 1B). The LBDs bind agonists, coagonists, competitive antagonists, and allosteric modulators to control gating properties of the ion channel pore formed by the TMD. The ATD regulates channel properties such as open probability and speeds of activation and deactivation (Gielen et al., 2009; Yuan et al., 2009), and also binds to allosteric modulators to either potentiate or inhibit the channel activities (Williams, 1993, 1997; Karakas et al., 2011) in a subtype-specific manner.

Our recent crystallographic study on the GluN1-GluN2A LBD unraveled the binding pattern of two antagonists, D-AP5 and PPDA (Jespersen et al., 2014). This study showed that the $\alpha$-carboxylate group contained in both of these compounds interacts with a conserved arginine residue (Arg518 in GluN2A), whereas distinct chemical moieties of D-AP5 and PPDA occupy different subsites, which we named Site I and Site II (Jespersen et al., 2014). Specifically, Site I accommodates the phosphono group of D-AP5 via direct polar interactions, and Site II binds to the phenanthrene group of PPDA mainly via hydrophobic interactions. A compound that does not occupy Site I, such as PPDA, can also bind and inhibit $\alpha$-amino-3-hydroxy-5-methyl-4-isoxazole propionic acid (AMPA) receptors and kainate receptors, indicating that the interaction between Site I and the phosphono group is a critical determinant for the specificity toward NMDA receptors. NVP-AAM077 has a unique bromophenyl substitution at the amino group nitrogen, which led us to hypothesize that the binding mode for NVP-AAM077 may be distinct from the ones for D-AP5 and PPDA. Structural studies on the intact NMDA receptor ion channels by X-ray crystallography and cryoelectron microscopy have shown patterns of conformational alterations linked to functions in multiple domains, which are associated with activation and inhibition (Karakas and
Furukawa, 2014; Lee et al., 2014; Tajima et al., 2016; Zhu et al., 2016). However, the structural studies on the intact NMDA receptors have not been ideal for monitoring exact compound binding modes at the atomic level due to their low resolution nature (3.6-15 $\mathrm{A}$ ). For this reason, in this study, we implemented X-ray crystallography on the isolated GluN1GluN2A LBD heterodimer, which is amenable to high-resolution structural analysis. We report in this work a crystal structure of the GluN1-GluN2A LBD complexed to NVP-AAM077 at 1.6 Å resolution. The structure shows a previously unobserved mode of antagonist binding involving residues from both GluN1 and GluN2A. Despite the unique binding mode, the NVP-AAM077 binding stabilizes the open-cleft conformation of the bi-lobed architecture of the GluN2A LBD in a similar manner to the D-AP5 and PPDA bindings. Additionally, the crystal structure also explains why NVP-AAM077 does not qualify as a GluN2A-specific antagonist.

\section{Materials and Methods}

Expression and Purification of GluN1 and GluN2A LBDs. The rat GluN1 LBD is defined as S1 (Met394 to Lys544) and S2 (Arg663 to Ser800) connected by a Gly-Thr linker and was expressed in OrigamiB (DE3) cells (Novagen, Darmstadt, Germany) under the T7 promoter in pET22b(+), as described previously (Furukawa and Gouaux, 2003; Jespersen et al., 2014). After growing cells to an optical density $_{600}=1.5$, protein expression was induced by $0.5 \mathrm{mM}$ isopropylbeta-D-1-thiogalactopyranoside for 22 hours at $15^{\circ} \mathrm{C}$. The protein was purified using nickel-nitrilotriacetic acid chelating Sepharose, followed by thrombin digestion to remove the polyhistidine tag. The digested product was purified by a SP-Sepharose column (GE Healthcare, Pittsburgh, PA). The rat GluN2A LBD composed of S1 (Asp402 to Arg539) and S2 (Gln661 to Asn802) connected by a Gly-Thr dipeptide linker was expressed as a fusion protein to small ubiquitin-like modifier with a hexa-histidine tag at the $\mathrm{N}$ terminus in OrigamiB (DE3) cells in the same way as for GluN1 LBD. Protein purification was conducted using nickel-nitrilotriacetic acid chelating Sepharose chromatography, followed by digestion by ubiquitin ligase protease-1 to remove small ubiquitin-like modifier. The digested product was further purified by Q-Sepharose and SP-Sepharose ion exchange chromatography (GE Healthcare), as described previously (Jespersen et al., 2014). 
Crystallography. Purified GluN1 and GluN2A LBD proteins were individually concentrated to $6 \mathrm{mg} / \mathrm{ml}$, mixed at a $1: 1(\mathrm{w} / \mathrm{w})$ ratio, and dialyzed against a buffer containing $10 \mathrm{mM}$ HEPES-NaOH $(\mathrm{pH}$ 7.0), $100 \mathrm{mM} \mathrm{NaCl}, 1 \mathrm{mM}$ L-glutamate, and $10 \mu \mathrm{M}$ glycine at $4^{\circ} \mathrm{C}$ overnight. The GluN1-GluN2A LBD crystals were produced by vapor diffusion at $18^{\circ} \mathrm{C}$ by the hanging drop method. Specifically, the purified proteins and the reservoir solution containing $18 \%$ polyethylene glycol monomethylether 2000, $100 \mathrm{mM}$ HEPES-NaOH $(\mathrm{pH}$ 7.0 ), and $75 \mathrm{mM} \mathrm{NaCl}$ were mixed at a $2: 1$ volume ratio and vapor diffused against the reservoir solution. Crystals appeared after 1 to 2 days. Crystals were then soaked against a buffer (100 mM HEPES, $\mathrm{pH} 7.0,75 \mathrm{mM} \mathrm{NaCl}, 18 \%$ polyethylene glycol monomethylether 2000 , and $1 \mathrm{mM}$ glycine) supplemented with $0.2 \mathrm{mM}$ NVP-AAM077 for 12 hours and with 0.4 mM NVP-AAM077 for another 5 hours. Finally, crystals were soaked against a buffer containing $1 \mathrm{mM}$ NVP-AAM077 and $18 \%$ glycerol for a few seconds and immediately flash frozen by liquid nitrogen. X-ray diffraction data were collect at the $\times 25$ beamline at the National Synchrotron Light Source at Brookhaven National Laboratory and processed using HKL2000 (Otwinowski and Minor, 1997). All of the structures were determined by molecular replacement using three search probes: GluN1 LBD, GluN2A LBD domain 1 (Asn404-Glu530 and Gly760-His801) and GluN2A LBD domain 2 (Thr531-Thr759) from the structural coordinate of the GluN1GluN2A LBD (Protein Data Bank code: 4NF8) (Jespersen et al., 2014). Rotational and translational search by the program PHASER (McCoy et al., 2007) resulted in the $Z$ scores, and Log-likelihood gain (LLG) values were rotation function Z-score $(\mathrm{RFZ})=20.7$, translation function Z-score $(T F Z)=28.0$, and LLG = 869 for GluN1 LBD; RFZ = 13 , $\mathrm{TFZ}=44.7$, and LLG $=2345$ for GluN2A LBD domain 2; and $\mathrm{RFZ}=11.9, \mathrm{TFZ}=51.5$, and $\mathrm{LLG}=4204$ for GluN2A LBD domain 2. Structural refinement and model building were performed with PHENIX (Adams et al., 2002) and Coot (Emsley and Cowtan, 2004), respectively. The degree of domain opening between the glutamatebound GluN2A LBD and the NVP-AAM077-bound GluN2A LBD was calculated using the program Pymol and running the python script,

\section{TABLE 1}

Data collection and refinement statistics

Values in parentheses are for the highest-resolution shell.

\begin{tabular}{|c|c|}
\hline & $\begin{array}{l}\text { GluN1-GluN2A ABD } \\
\text { NVP-AAM077 }\end{array}$ \\
\hline \multicolumn{2}{|l|}{ Data collection } \\
\hline Resolution $(\AA)$ & $50-1.6(1.66-1.60)$ \\
\hline Space Group & $\mathrm{P} 22_{1} 2_{1} 2_{1}$ \\
\hline \multicolumn{2}{|l|}{ Unit cell dimensions } \\
\hline $\mathrm{a}(\AA)$ & 59.550 \\
\hline$b(\AA)$ & 84.227 \\
\hline c $(\AA)$ & 121.218 \\
\hline Completeness (\%) & $89.5(55.3)$ \\
\hline$R_{\text {merge }}$ & $0.077(0.623)$ \\
\hline Redundancy & $4.3(1.6)$ \\
\hline $\mathrm{I} / / \sigma$ & $17.84(1.23)$ \\
\hline \multicolumn{2}{|l|}{ Refinement } \\
\hline No. of unique reflections & 72,823 \\
\hline $\mathrm{R}_{\mathrm{work}} / \mathrm{R}_{\text {free }}$ & $0.20 / 0.22$ \\
\hline \multicolumn{2}{|l|}{$\begin{array}{l}\mathrm{n}_{\text {work }} / \mathrm{n}_{\text {free }} \\
\text { No. atoms }\end{array}$} \\
\hline Protein & 4300 \\
\hline Compound & 27 \\
\hline Water & 253 \\
\hline Glycerol & 0 \\
\hline \multicolumn{2}{|l|}{$B$ factors } \\
\hline Protein & 22.34 \\
\hline Compound & 16.41 \\
\hline Water & 25.64 \\
\hline Glycerol & - \\
\hline \multicolumn{2}{|l|}{ RMSD } \\
\hline Bond lengths $(\AA)$ & 0.008 \\
\hline Bond angles $\left({ }^{\circ}\right)$ & 1.101 \\
\hline
\end{tabular}

RMSD, root-mean-square deviation distance. draw_rotation_axis.py (code written by Pablo Guardado Calvo downloaded from the pymolwiki website). Specifically, the C $\alpha$ s of the D1 regions were first superimposed by the super command in Pymol. The transformation matrix to superpose the $\mathrm{C} \alpha$ s of the D2 regions was calculated and used to generate a rotation axis.

Electrophysiology. Recombinant GluN1-1a/GluN2A NMDA receptors were expressed by coinjecting $0.1-0.5 \mathrm{ng}$ of the wild-type or mutant rat GluN1-1a and GluN2A cRNAs at a 1:1 ratio (w/w) into defolliculated Xenopus laevis oocytes. The two-electrode voltageclamp recordings were performed using agarose-tipped microelectrodes (0.4-1.0 M $\Omega$ ) filled with $3 \mathrm{M} \mathrm{KCl}$ at a holding potential of $-60 \mathrm{mV}$. The bath solution contained $5 \mathrm{mM}$ HEPES, $100 \mathrm{mM} \mathrm{NaCl}$, and $0.3 \mathrm{mM} \mathrm{BaCl}_{2}$, at $\mathrm{pH} 7.4$ (adjusted with potassium hydroxide). Currents were evoked by applications of $100 \mu \mathrm{M}$ glycine and various concentrations of L-glutamate. For analyzing inhibition by NVPAAM077, various concentrations of antagonists were applied in the presence of $5 \mu \mathrm{M}$ L-glutamate. The data sets were acquired and analyzed by the program Pulse (HEKA, Holliston, MA). Data points were plotted and fitted to calculate $\mathrm{EC}_{50}$ values of L-glutamate and $\mathrm{IC}_{50}$ values of antagonists using the program Kaleidagraph (Synergy Software). $K_{i}$ values were calculated with the Cheng-Prusoff equation: $K_{i}=\mathrm{IC}_{50} /\left(1+[\right.$ L-glutamate $\left.] / \mathrm{EC}_{50}\right)$.

\section{Results}

Structural Study of GluN1-GluN2A LBD Complexed to NVP-AAM077. To understand the binding mode and the resulting effect on the overall protein conformation, we attempted to obtain the high-resolution structure of the isolated LBD proteins. Toward this end, we took advantage of our previously established method for effectively obtaining high-resolution structures of the GluN1-GluN2A LBD complexed to antagonists (Jespersen et al., 2014). Specifically, the crystals of the GluN1-GluN2A LBD proteins were grown in the presence of glycine and glutamate and soaked against the crystallization buffer (see Materials and Methods) containing glycine and NVP-AAM077. Thus, in the crystal structure, the coagonist glycine is bound to the GluN1 LBD and the antagonist NVP-AAM077 is bound to the GluN2A LBD. Our crystals gave rise to X-ray diffraction to the Bragg spacing of $1.6 \AA$ (Table 1). The electron density was visible for 283 residues of the 289 residues derived from GluN1 and all of the 280 residues derived from GluN2A. The quality of the electron density was especially high at the GluN1-GluN2A subunit interface and at the NVP-AAM077 binding site, which allowed us to monitor the precise binding mode of NVP-AAM077 and the hydrogen bond network around the binding site.

Overall Architecture of NVP-AAM077-GluN1-GluN2A LBD. Both the GluN1 and GluN2A LBDs have the bi-lobed clamshell-like architecture composed of an upper domain (D1) and a lower domain (D2), as previously observed (Figs. 1 and 2) (Furukawa and Gouaux, 2003; Furukawa et al., 2005; Jespersen et al., 2014). The GluN1 and GluN2A LBD proteins crystallized as a heterodimer as in the previous case (Jespersen et al., 2014). This heterodimeric subunit arrangement is physiologic as the similar GluN1-GluN2A LBD heterodimer is observed in the structure of the intact heterotetrameric NMDA receptor ion channels (Karakas and Furukawa, 2014; Lee et al., 2014). The main locus for the NVPAAM077 binding is the D1-D2 domain cleft in the GluN2A subunit where the neurotransmitter agonist, glutamate, also binds. Furthermore, the bromophenyl group of NVP-AAM077 extends toward the GluN1 subunit (Fig. 2B) and makes an 
A

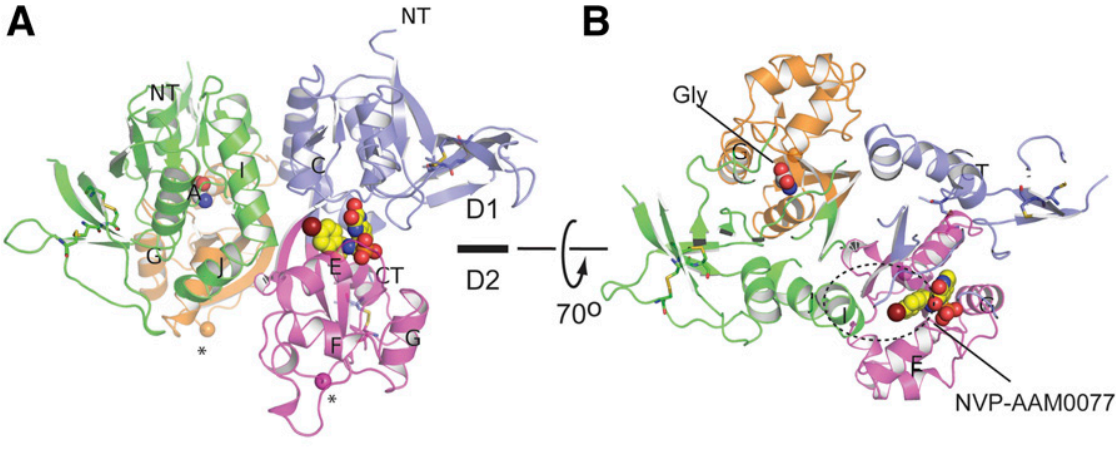

GluN1 (gly) - GluN2A (NVP-AAM0077)
Fig. 2. Crystal structure of NVP-AAM077-GluN1GluN2A LBD. (A) The crystal structure of the GluN1GluN2A LBD complexed to glycine (Gly, light gray spheres) and NVP-AAM077 (yellow spheres) to GluN1 (green and orange) and GluN2A (purple and magenta), respectively, viewed from the side of the $\mathrm{N}$ terminus (NT) and the C-terminus (CT). Spheres with asterisks are the $\mathrm{C} \alpha$ s of the Gly-Thr linker. Cysteine residues forming disulfide bonds are shown as sticks. The LBDs have a clamshell-like architecture composed of two bi-lobe domains, D1 (green in GluN1 and purple in GluN2A) and D2 (orange in GluN1 and magenta in GluN2A). (B) The same structure viewed from the top of the $\mathrm{N}$ termini. The bromophenyl group of NVP-AAM077 extends toward Helix I of the GluN1 subunit (dotted oval). Assigned letters for alpha helices are in accordance with Jespersen et al, 2014. additional contact with a GluN1 residue, as described in more detail in the following sections.

Comparison with GluN1-GluN2A LBD Bound to Glutamate and Other Antagonists. Binding of NVPAAM077 to the GluN1-GluN2A LBD results in the rigidbody opening of the D1-D2 bi-lobe in GluN2A compared with that bound to glutamate (Fig. 3). Stabilization of the open conformation upon binding of competitive antagonists is a feature previously observed in the structural studies of the GluN1-GluN2A LBD complexed to D-AP5 and (-)-PPDA (Jespersen et al., 2014). The degree of the bi-lobe opening is $\sim 16^{\circ}, \sim 21^{\circ}$, and $\sim 17^{\circ}$ for D-AP5, PPDA, and NVP-AAM077, respectively (Fig. 3B; Materials and Methods). Thus, the extent of bi-lobe opening in the NVP-AAM077-bound GluN2A LBD is more similar to that of the D-AP5-bound GluN2A LBD than the PPDA-bound GluN2A LBD.

The opening and closing motions of the LBD bi-lobes are considered important for controlling ion channel gating in iGluRs. In NMDA receptors, the closure of the bi-lobes by agonist binding is suggested to move the LBD-TMD linkers of the GluN1 and GluN2A subunits apart to create a tension that is required to move the pore-forming M3 transmembrane helices (Kazi et al., 2014). Contrary to agonist binding, antagonist binding stabilizes the LBD bi-lobes in an open conformation, thereby keeping the distance between the LBD-TMD linkers of GluN1 and GluN2A sufficiently short. This prevents formation of tensions; thus, the TMD ion channels remain closed (Fig. 3). The residues GluN1Ile664 and GluN2A-Val662 are located at the upper edge of the LBD-TMD linker and are subject to movement upon ligand binding (Fig. 3C). Upon binding of NVP-AAM077 to GluN2A, the distance between GluN1-Ile664 and GluN2AVal662 shortens by $3.9 \AA$ compared with that when glutamate is bound. Contrary to the GluN2A LBD, there is little or no change in the GluN1 subunit between the glutamate-bound and NVP-AAM077-bound GluN1-GluN2A LBD as represented by the low root-mean-square deviation distance (0.53 $\AA$ over $275 \mathrm{C} \alpha$ positions). No apparent change was observed in the pattern of the GluN1-GluN2A subunit arrangement.
A

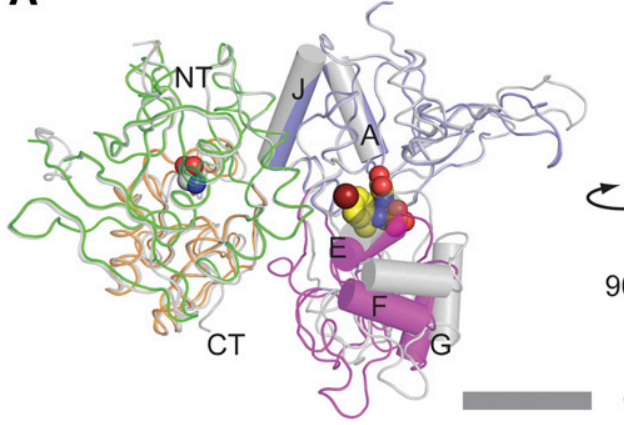

GluN1

GluN2A

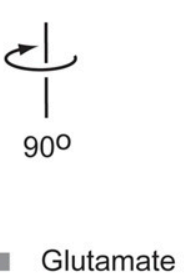

NVP-AAM077
B

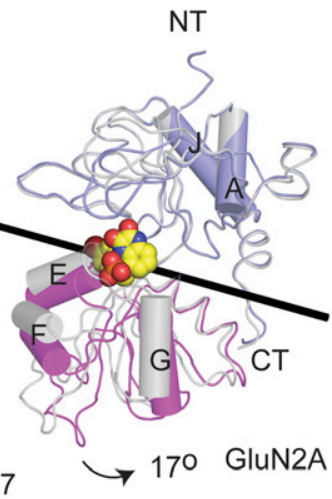

C

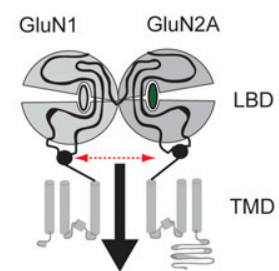

GluN1/GluN2A

Glycine/Glutamate

(Open Channel)

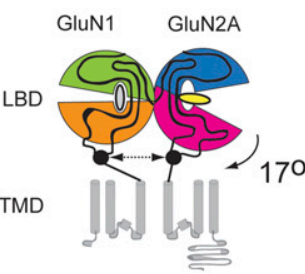

GluN1/GluN2A

glycine/NVP-AAM077

(Closed Channel)
Fig. 3. Comparison of NVP-AAM077-GluN1-GluN2A LBD with glu-GluN1-GluN2A LBD. (A) Comparison of the GluN1-GluN2A heterodimers by superposition of the D1 of the GluN2A LBDs bound to glutamate (Protein Data Bank code: 4NF8) and NVP-AAM077. The color code for the NVP-AAM077-GluN1-GluN2A LBD is the same as in Fig. 1. The structure of the glutamate-bound GluN1-GluN2A LBD (glu-GluN1GluN2A LBD) is in gray. The structure is viewed from the side of the $\mathrm{N}$-terminus (NT) and the C-terminus (CT). Assigned letters for alpha helices are in accordance with Jespersen et al, 2014. (B) The same superposed structures viewed from a different angle. Only GluN2A LBD is shown here for clarity. The rotational angle to superpose $\mathrm{C} \alpha \mathrm{s}$ of $\mathrm{D} 2$ is calculated to be $17^{\circ}$ around the pivotal axis shown as a black rod, which indicates that the NVP-AAM077-bound GluN2A LBD is $17^{\circ}$ more open than the glutamatebound GluN2A LBD. No significant change was observed in GluN1 LBDs between the two structures. (C) Schematic illustration to show that the domain opening in GluN2A reduces the distance between the LBDTMD linkers of GluN1 and GluN2A subunits, thereby reducing the tension necessary for channel gating. 

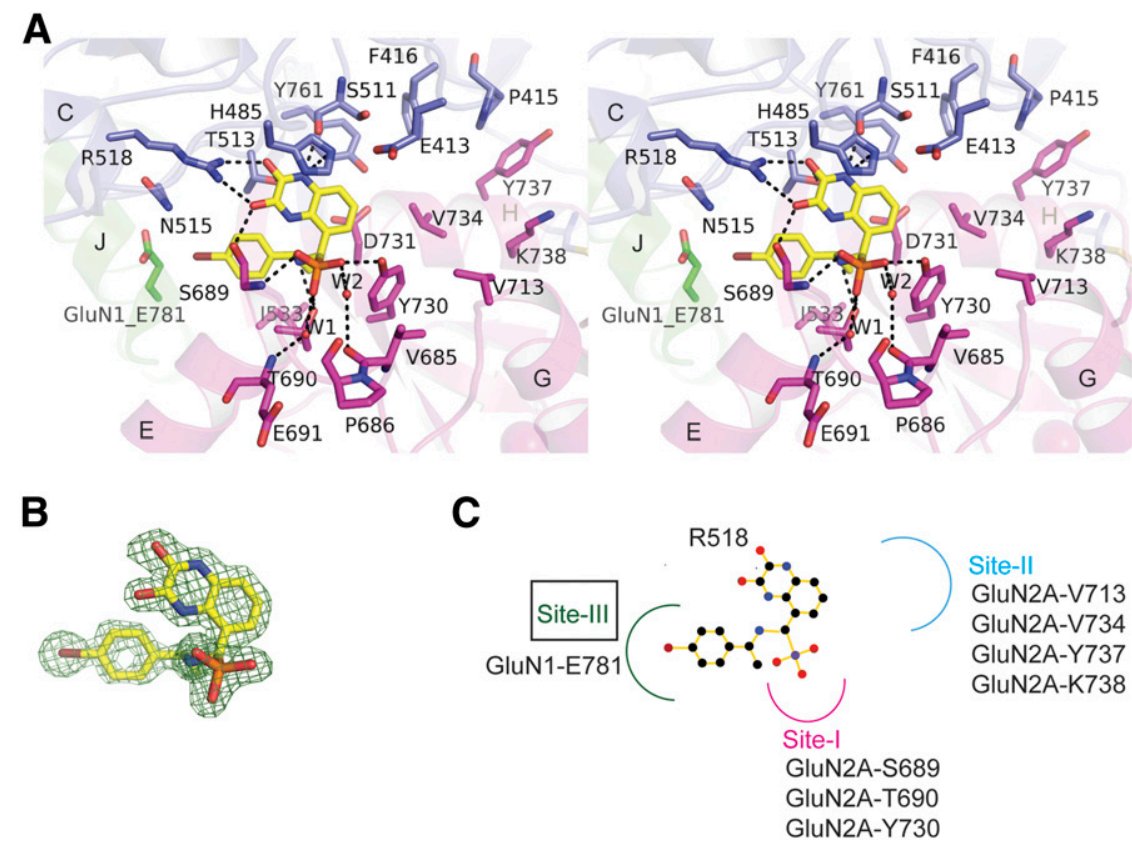

Fig. 4. Binding site of NVP-AAM077. (A) Stereoview of the NVP-AAM077 binding site. The binding of NVP-AAM077 (yellow sticks) is mediated by a number of polar interactions and a halogen interaction (black dashed lines). The carboxyl side chain of GluN1-Glu781 (green sticks) interacts with the bromine atom in the bromophenyl group of NVPAAM077 via a halogen bond. (B) The Fo-Fc omit map of NVP-AAM077 contoured at $3 \sigma$ illustrates clear density for every chemical feature of the compound. (C) Schematic illustration of the NVPAAM077 binding site. The phosphono group and the bromophenyl group occupy Site I and Site III, respectively, whereas no chemical group binds to Site II. Helices are labelled as in Fig. 2 and 3.

Novel Binding Mode of NVP-AAM077. The $1.6 \AA$ resolution structure from the current crystallographic study provided an accurate map of the compound binding site (Fig. 4; Table 1). Substitution of glutamate with NVP-AAM077 was near complete as there is no visual evidence for the remaining glutamate density after extensive crystal soaking. Our previous studies revealed elements of the binding pocket important for recognition of D-AP5 and PPDA (Jespersen et al., 2014). The phosphono group in D-AP5 occupies the subsite, Site I, consisting of polar residues including GluN2ASer689, GluN2A-Thr690, and GluN2A-Tyr730, whereas the phenanthrene group in PPDA occupies the subsite, Site II,

A

GIUN

GluN2A

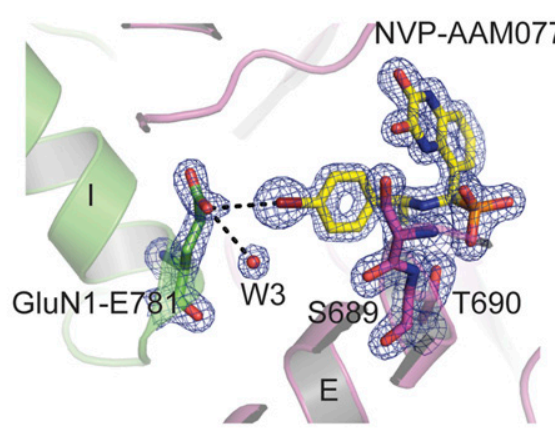

C

GluN1

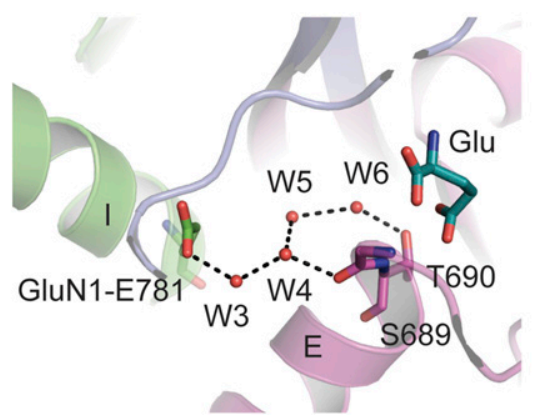

B

B GluN1 GluN2A

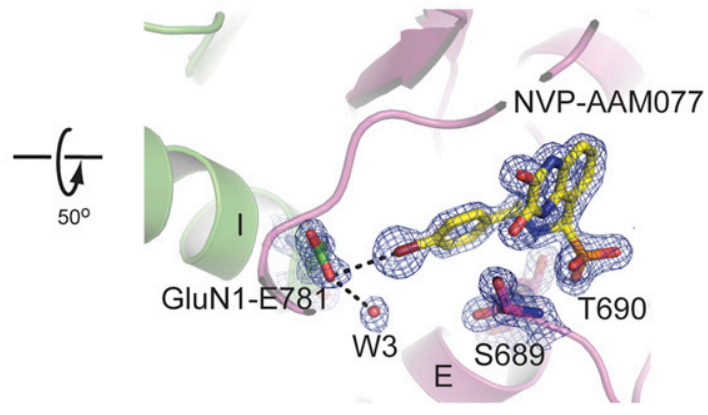

D
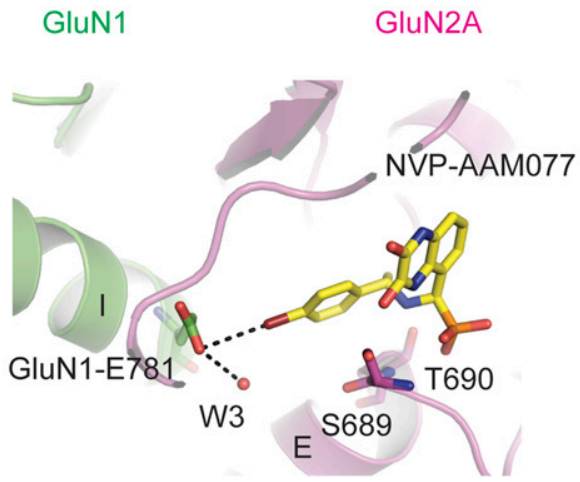

Fig. 5. NVP-AAM077 forms halogen bond with GluN1-Glu781. (A and B) The $2 F_{O}-F_{C}$ map contoured at $1.5 \sigma$ shows ordered electron density for the residue GluN1-Glu781, a water molecule W3, and NVP-AAM077. Electron density for other residues and water molecules is omitted for clarity. (C) The GluN1-GluN2A subunit interface in the glutamate-bound GluN1-GluN2A LBD structure (Protein Data Bank code: 4 NF8). (D) The same interface in the NVP-AAM077-bound GluN1-GluN2A LBD structure. The placement of the bromophenyl group displaces three water molecules (W4-6) that are forming intersubunit interactions between GluN1Glu781 and GluN2A-Ser689 or GluN2AThr690. The distance between the carboxyl oxygen in GluN1-Glu781 and the bromine atom in the bromophenyl group is $3.5 \AA$ in this structure. The helices are labelled as in Fig. 2, 3, and 4. 

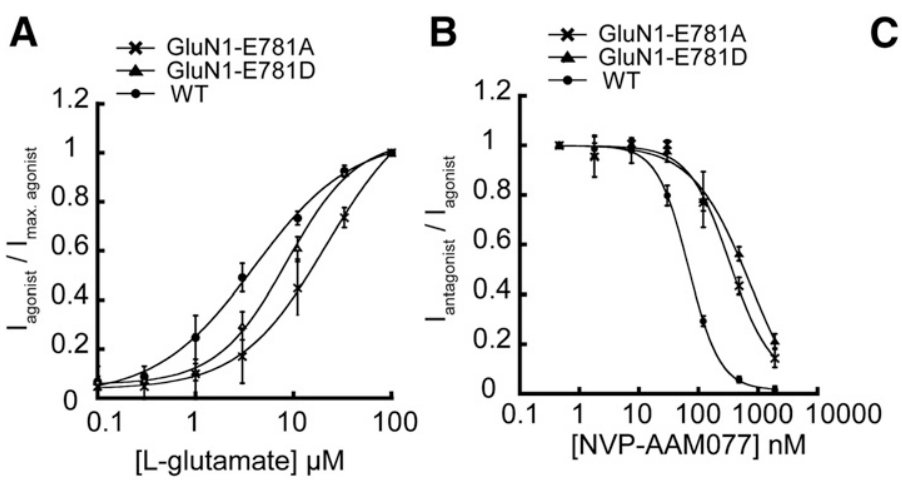

consisting of hydrophobic residues (Jespersen et al., 2014). Binding of NVP-AAM077 is mediated by a number of direct interactions, including polar interactions between the dioxoquinoxalinyl group and residues from D1 (GluN2A-Ser511, -Thr513, and -Arg518), the phosphono group and residues from Site I, and the bromophenyl group and GluN1-Glu781 at Site III (Fig. 4C). The extension of the bromophenyl group toward the GluN1-GluN2A subunit interface and the direct interaction of the bromine atom with the carboxylate group of GluN1-Glu781 at Site III is a binding mode previously unobserved. The placement of the bromophenyl group at Site III displaces three water molecules present in the glutamate-GluN1-GluN2A LBD, which are involved in water-mediated polar interactions that tether GluN1-Glu781 and GluN2A-Ser689 or GluN2A-Thr690, thereby stabilizing the intersubunit interaction in addition to the opening of the GluN2A bi-lobe (Fig. 5). The distance between the bromine atom from NVP-AAM077 and the carboxylate group of GluN1-Glu781 is $3.5 \AA$, which slightly deviates from the ideal distance for bond formation (suggested to be $3.37 \AA$ for a bromine-oxygen interaction) (Auffinger et al., 2004; Sirimulla et al., 2013). We speculate that a weak bromine-oxygen interaction exists between GluN1-Glu781 and a bromine atom because the electron density for the bromophenyl group and the GluN1-Glu781 is highly ordered (Fig. 5, A and B). Other key interactions between NVP-AAM077 and the GluN1GluN2A LBD include polar interactions between the dioxoquinoline group and GluN2A-Ser689, the amino-bromophenyl group and GluN2A-Thr690, and water-mediated hydrogen bonds involving GluN2A-Val685, -Pro686, -Gly688, and -Glu691 (W1 and W2; Fig. 4A).

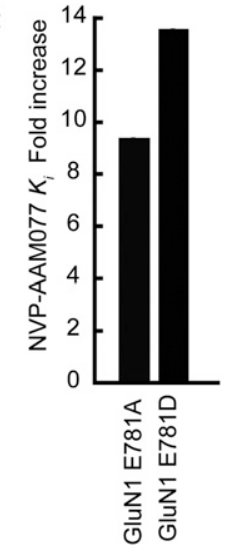

Fig. 6. Validation of NVP-AAM077-GluN1 interaction (A) Mutational analysis of the mutant channels containing GluN1-Glu781Ala or GluN1-Glu781Asp and the wild-type (WT) GluN2A by two-electrode voltage clamp (TEVC). Dose response of glutamate was measured in the presence of $100 \mu \mathrm{M}$ glycine. (B) Inhibition of the NMDA receptor currents by various concentrations of NVP-AAM007 in the presence of $100 \mu \mathrm{M}$ glycine and $5 \mu \mathrm{M}$ glutamate. (C) $K_{\mathrm{i}}$ values were calculated with the Cheng-Prusoff equation, as described in the Materials and Methods section and compared with that of the wild-type channel. Data points were acquired from independent recording using six different Xenopus oocytes. Error bars represent S.D.
Validation of NVP-AAM077-Binding Mode. To validate the physiologic relevance of the new antagonist-binding mode observed in the NVP-AAM077-GluN1-GluN2A LBD structure, we conducted site-directed mutagenesis coupled with two-electrode voltage clamp. Although the binding pattern in Site I is similar between the NVPAAM077- and D-AP5-bound structures, the compound recognition by Site III at the GluN1-GluN2A LBD interface is unprecedented (Figs. 4 and 5). Thus, we focused on validating the importance of Site III by conducting site-directed mutagenesis of GluN1-Glu781, which interacts with the bromine atom in the bromophenyl group of NVP-AAM077. We mutated GluN1-Glu781 to aspartate or alanine to alter the distance between the carboxylate group and the bromine atom or to remove the carboxylate group, respectively. We estimated $K_{\mathrm{i}}$ values by measuring the $\mathrm{EC}_{50}$ of glutamate and $\mathrm{IC}_{50}$ of NVP-AAM077 (Fig. 6; Supplemental Fig. 1). As predicted by the presence of the water-mediated hydrogen bond network in Site III in the glutamate-GluN1-GluN2A LBD structure (Fig. 5C), we saw changes in the glutamate potency when this network was broken by the GluN1-Glu781Asp and the GluN1-Glu781Ala mutations, indicating the importance of the subunit interface in activity (Fig. 6A). Nevertheless, the $K_{\mathrm{i}}$ values for NVP-AAM077 in the GluN1Glu781Asp and -Glu781Ala mutants were greater by $\sim 13$-fold and ninefold compared with the wild type, respectively (Fig. 6, B and C; Table 2). Thus, the mutagenesis result indicated that the interaction between the bromophenyl group in NVP-AAM077 and GluN1Glu781 is critical for inhibition.

TABLE 2

Potency of NVP-AAM077

$\mathrm{IC}_{50}$ values were calculated in the presence of $5 \mu \mathrm{M}$ glutamate and $100 \mu \mathrm{M}$ glycine for all of the mutants. Kivalues were calculated by the Cheng-Prusoff equation: $\boldsymbol{K}_{i}=\boldsymbol{I C}_{50} /\left(1+\left\{[\boldsymbol{L}\right.\right.$ - glutamate $\left.] \boldsymbol{E C _ { 5 0 } \}}\right) \mathrm{EC}_{50}$ and $\mathrm{IC}_{50} \pm$ S.D. represents values from three and six different oocytes per experiment, respectively. \pm S.D. for Ki and Ki-fold was calculated with the error propagation formula as follows: $(\delta \boldsymbol{Q} / \boldsymbol{Q})=\sqrt{(\delta \boldsymbol{d} / \boldsymbol{a})^{2}+\ldots+(\delta z / z)^{2}}$

\begin{tabular}{lrcrr}
\hline & Glutamate $\mathrm{EC}_{50} \mu \mathrm{M}$ & ${\text { NVP-AAM077 } \mathrm{IC}_{50} \mathrm{nM}}$ & NVP-AAM077 $K_{i} \mathrm{nM}$ & $\begin{array}{c}\text { NVP-AAM077 } \\
K_{i} \text {-Fold } \\
\text { Increase }\end{array}$ \\
\hline GluN1-GluN2A WT & $3.81 \pm 0.89$ & $68.40 \pm 3.79$ & $29.58 \pm 0.24$ & - \\
GluN1 E781D & $8.55 \pm 1.54$ & $636.9 \pm 79.35$ & $401.93 \pm 0.21$ & $13.58 \pm 0.008$ \\
GluN1 E781A & $20.85 \pm 3.39$ & $344.47 \pm 42.94$ & $277.84 \pm 0.20$ & $9.39 \pm 0.008$ \\
\hline
\end{tabular}




\section{Discussion}

The current high-resolution structural study revealed a novel antagonist-binding mode of NVP-AAM077. Specifically, the bromophenyl group of NVP-AAM077 extends out of the glutamate-binding pocket in GluN2A toward the GluN1GluN2A subunit interface, and interacts with GluN1-Glu781. This marks the first example in which a competitive antagonist for NMDA receptors is recognized by residues from both GluN1 and GluN2 subunits.

The subunit interfaces in iGluRs are generally known to be critical for regulating activities of their oligomeric ion channels (Karakas et al., 2015; Regan et al., 2015). Some of the subunit interfaces in iGluRs also serve as binding sites for compounds for functional regulation. Such an example in NMDA receptors is an allosteric inhibitor, ifenprodil, which binds to the GluN1-GluN2B subunit interface at ATD (Karakas et al., 2011). Other examples are binding of negative allosteric modulator (NAM) and positive allosteric modulator compounds, which have been shown to occur at the GluN1GluN2A LBD subunit interface by recent structural studies (Hackos et al., 2016; Yi et al., 2016). Even though the molecular mechanism is yet to be determined clearly, these studies indicated that stabilization of the subunit interface can upregulate or downregulate the ion channel activity. In AMPA receptors, the subunit interface of the LBD homodimer accommodates cyclothiazide and aniracetam, which alleviate desensitization and slow the speed of deactivation (Sun et al., 2002; Jin et al., 2005). The stabilization of the subunit interface by a mutation or artificial disulfide cross-link in AMPA receptors has also been shown to reduce desensitization, indicating that the strength of the intersubunit interaction in AMPA receptors controls the ion channel function (Stern-Bach et al., 1998; Weston et al., 2006). Of note is the fact that there is no positional overlap between binding sites of NVP-AAM077 and the NMDA receptor NAMs; thus, the inhibitory effect of NVP-AAM077 is most likely distinct from that of NAM even though a portion of NVP-AAM077 resides at the subunit interface.

Binding of NVP-AAM077 results in opening of the bi-lobe of the GluN2A LBD compared with the glutamate-bound GluN2A LBD. A similar pattern of rigid body domain movement was previously observed in the GluN1-GluN2A LBD bound to D-AP5 and PPDA (Jespersen et al., 2014). The recent structural study on the intact GluN1-GluN2B NMDA receptors solved in the presence of antagonists for both GluN1 and GluN2 subunits, 5,7-dichlorokynurenic acid and D-AP5, showed extensive disruption of the LBD subunit interface where NAM, positive allosteric modulator, and NVP-AAM077 bind (Zhu et al., 2016). In the current and previous crystallographic studies, such disruption in the subunit interface did not occur. There are several potential reasons for this different outcome. First, our current study on NVP-AAM077 was conducted in the presence of the coagonist glycine bound to the GluN1 LBD. Second, our study is conducted in the absence of ATD or TMD; thus, the GluN1-GluN2A LBD is unaffected by conformational movements of these other domains (RomeroHernandez et al., 2016; Tajima et al., 2016). Third, crystal contacts may be restricting the spatial freedom to potentially disrupt the subunit interaction. Last, the effect of antagonist binding on the overall protein conformation may be different between the GluN1-GluN2A and GluN1-GluN2B NMDA receptors. Nevertheless, our high-resolution structure, along with our electrophysiological assays, clearly indicated that the NVP-AAM077 binding occurs partially at the GluN1-GluN2A subunit interface. Our structural observation indicates that the inhibitory effect of NVP-AAM077 is mostly occurring via opening of the GluN2A LBD bi-lobe as observed in the structural studies of the GluN1-GluN2A LBD complexed to D-AP5 and PPDA (Jespersen et al., 2014). Opening of the GluN2A LBD bi-lobe most likely relieves the tension in the LBD-TMD linker (Kazi et al., 2014), thereby preventing channel opening by interfering with a transduction of the physical force from the LBDs to the TMD.

NVP-AAM077 was originally pursued with enthusiasm due to its reported specificity toward the GluN2A-containing NMDA receptors. The extensive study to characterize the NVP-AAM077 pharmacology later showed that the degree of specificity is minimal (Frizelle et al., 2006). Our structural study shows that there is no apparent structural element that qualifies NVP-AAM077 as a GluN2A-specific antagonist, as residues from Site I and Site III are completely conserved. We expect Site III in the GluN1-GluN2B subunit interface to be almost identical to the GluN1-GluN2A subunit interface because the subunit arrangement between the GluN1GluN2A LBD and the GluN1-GluN2B LBD is almost identical (Karakas and Furukawa, 2014). Thus, our study supports little or no subtype specificity of NVP-AAM077. Nevertheless, the new antagonist-binding mode highlighted by the newly observed Site III provides an opportunity to pursue novel compound designs.

\section{Acknowledgments}

The authors thank staff at the X25 beamline at the National Synchrotron Light Source in the Brookhaven National Laboratory. Erkan Karakas is thanked for help in crystallography. The structural coordinates related to this work have been deposited to the Protein Data Bank with the entry code 5U8C.

\section{Authorship Contributions}

Participated in research design: Romero-Hernandez, Furukawa. Conducted experiments: Romero-Hernandez, Furukawa.

Performed data analysis: Romero-Hernandez, Furukawa.

Wrote or contributed to the writing of the manuscript: RomeroHernandez, Furukawa.

\section{References}

Adams PD, Grosse-Kunstleve RW, Hung LW, Ioerger TR, McCoy AJ, Moriarty NW, Read RJ, Sacchettini JC, Sauter NK, and Terwilliger TC (2002) PHENIX: building new software for automated crystallographic structure determination. Acta Crystallogr D Biol Crystallogr 58:1948-1954.

Auberson YP, Allgeier H, Bischoff S, Lingenhoehl K, Moretti R, and Schmutz M (2002) 5-Phosphonomethylquinoxalinediones as competitive NMDA receptor antagonists with a preference for the human $1 \mathrm{~A} / 2 \mathrm{~A}$, rather than $1 \mathrm{~A} / 2 \mathrm{~B}$ receptor composition. Bioorg Med Chem Lett 12:1099-1102.

Auffinger P, Hays FA, Westhof E, and Ho PS (2004) Halogen bonds in biological molecules. Proc Natl Acad Sci USA 101:16789-16794.

Emsley P and Cowtan K (2004) Coot: model-building tools for molecular graphics. Acta Crystallogr D Biol Crystallogr 60:2126-2132.

Evans RH, Francis AA, Jones AW, Smith DA, and Watkins JC (1982) The effects of a series of omega-phosphonic alpha-carboxylic amino acids on electrically evoked and excitant amino acid-induced responses in isolated spinal cord preparations. $\mathrm{Br} J$ Pharmacol 75:65-75.

Feng B, Tse HW, Skifter DA, Morley R, Jane DE, and Monaghan DT (2004) Structure-activity analysis of a novel NR2C/NR2D-preferring NMDA receptor antagonist: 1-(phenanthrene-2-carbonyl) piperazine-2,3-dicarboxylic acid. $\mathrm{Br} J$ Pharmacol 141:508-516.

Frizelle PA, Chen PE, and Wyllie DJ (2006) Equilibrium constants for (R)-[(S)-1-(4bromo-phenyl)-ethylamino]-(2,3-dioxo-1,2,3,4-tetrahydroquinoxalin-5-yl)-methyl]phosphonic acid (NVP-AAM077) acting at recombinant NR1/NR2A and NR1/NR2B $\mathrm{N}$-methyl-D-aspartate receptors: implications for studies of synaptic transmission. Mol Pharmacol 70:1022-1032. 
Furukawa H and Gouaux E (2003) Mechanisms of activation, inhibition and specificity: crystal structures of the NMDA receptor NR1 ligand-binding core. EMBO $J$ 22:2873-2885.

Furukawa H, Singh SK, Mancusso R, and Gouaux E (2005) Subunit arrangement and function in NMDA receptors. Nature 438:185-192.

Gielen M, Siegler Retchless B, Mony L, Johnson JW, and Paoletti P (2009) Mechanism of differential control of NMDA receptor activity by NR2 subunits. Nature 459:703-707.

Hackos DH, Lupardus PJ, Grand T, Chen Y, Wang TM, Reynen P, Gustafson A, Wallweber HJ, Volgraf M, Sellers BD, et al. (2016) Positive allosteric modulators of GluN2A-containing NMDARs with distinct modes of action and impacts on circuit function. Neuron 89:983-999.

Jespersen A, Tajima N, Fernandez-Cuervo G, Garnier-Amblard EC, and Furukawa H (2014) Structural insights into competitive antagonism in NMDA receptors. Neuron 81:366-378.

Jin R, Clark S, Weeks AM, Dudman JT, Gouaux E, and Partin KM (2005) Mechanism of positive allosteric modulators acting on AMPA receptors. $J$ Neurosci 25 9027-9036

Karakas E and Furukawa H (2014) Crystal structure of a heterotetrameric NMDA receptor ion channel. Science 344:992-997.

Karakas E, Regan MC, and Furukawa H (2015) Emerging structural insights into the function of ionotropic glutamate receptors. Trends Biochem Sci 40:328-337.

Karakas E, Simorowski N, and Furukawa H (2011) Subunit arrangement and phenylethanolamine binding in GluN1/GluN2B NMDA receptors. Nature 475: 249-253.

Kazi R, Dai J, Sweeney C, Zhou HX, and Wollmuth LP (2014) Mechanical coupling maintains the fidelity of NMDA receptor-mediated currents. Nat Neurosci 17: 914-922.

Lee CH, Lü W, Michel JC, Goehring A, Du J, Song X, and Gouaux E (2014) NMDA receptor structures reveal subunit arrangement and pore architecture. Nature 511:191-197.

McCoy AJ, Grosse-Kunstleve RW, Adams PD, Winn MD, Storoni LC, and Read RJ (2007) Phaser crystallographic software. J Appl Cryst 40:658-674.

McLennan H and Lodge D (1979) The antagonism of amino acid-induced excitation of spinal neurones in the cat. Brain Res 169:83-90.

Otwinowski Z and Minor W (1997) Processing of x-ray diffraction data collected in oscillation mode. Methods Enzymol 276:307-326.

Regan MC, Romero-Hernandez A, and Furukawa H (2015) A structural biology perspective on NMDA receptor pharmacology and function. Curr Opin Struct Biol 33:68-75.
Romero-Hernandez A, Simorowski N, Karakas E, and Furukawa H (2016) Molecular basis for subtype specificity and high-affinity zinc inhibition in the GluN1-GluN2A NMDA receptor amino-terminal domain. Neuron 92:1324-1336.

Sirimulla S, Bailey JB, Vegesna R, and Narayan M (2013) Halogen interactions in protein-ligand complexes: implications of halogen bonding for rational drug design. $J$ Chem Inf Model 53:2781-2791.

Stern-Bach Y, Russo S, Neuman M, and Rosenmund C (1998) A point mutation in the glutamate binding site blocks desensitization of AMPA receptors. Neuron 21: 907-918.

Sun Y, Olson R, Horning M, Armstrong N, Mayer M, and Gouaux E (2002) Mechanism of glutamate receptor desensitization. Nature 417:245-253.

Tajima N, Karakas E, Grant T, Simorowski N, Diaz-Avalos R, Grigorieff N, and Furukawa $\mathrm{H}$ (2016) Activation of NMDA receptors and the mechanism of inhibition by ifenprodil. Nature 534:63-68.

Traynelis SF, Wollmuth LP, McBain CJ, Menniti FS, Vance KM, Ogden KK, Hansen KB, Yuan H, Myers SJ, and Dingledine R (2010) Glutamate receptor ion channels: structure, regulation, and function. Pharmacol Rev 62:405-496.

Weston MC, Schuck P, Ghosal A, Rosenmund C, and Mayer ML (2006) Conformational restriction blocks glutamate receptor desensitization. Nat Struct Mol Biol 13:1120-1127.

Williams K (1993) Ifenprodil discriminates subtypes of the N-methyl-D-aspartate receptor: selectivity and mechanisms at recombinant heteromeric receptors. $\mathrm{Mol}$ Pharmacol 44:851-859.

Williams K (1997) Interactions of polyamines with ion channels. Biochem $J$ 325: 289-297.

Yi F, Mou TC, Dorsett KN, Volkmann RA, Menniti FS, Sprang SR, and Hansen KB (2016) Structural basis for negative allosteric modulation of GluN2A-containing NMDA receptors. Neuron 91:1316-1329.

Yuan H, Hansen KB, Vance KM, Ogden KK, and Traynelis SF (2009) Control of NMDA receptor function by the NR2 subunit amino-terminal domain. $J$ Neurosci 29:12045-12058.

Zhu S, Stein RA, Yoshioka C, Lee CH, Goehring A, Mchaourab HS, and Gouaux E (2016) Mechanism of NMDA receptor inhibition and activation. Cell 165:704-714.

Address correspondence to: Dr. Hiro Furukawa, One Bungtown Road, Cold Spring Harbor, NY 11724. E-mail: furukawa@cshl.edu 